\title{
Infinite horizon optimal control problems with multiple thermostatic hybrid dynamics
}

\author{
Fabio Bagagiolo* and Katia Danieli
}

\begin{abstract}
We study an optimal control problem for a hybrid system exhibiting several internal switching variables whose discrete evolutions are governed by some delayed thermostatic laws. By the dynamic programming technique we prove that the value function is the unique viscosity solution of a system of several Hamilton-Jacobi equations, suitably coupled. The method involves a contraction principle and some suitably adapted results for exit-time problems with discontinuous exit cost.
\end{abstract}

Keywords: thermostatic switching systems, optimal control, infinite horizon, exit-time problems, Hamilton-Jacobi, viscosity solutions.

\section{Introduction}

In this paper we address the following model problem of optimal control. We consider the following hybrid controlled system

$$
\left\{\begin{array}{l}
y^{\prime}(t)=f\left(y(t), w_{1}(t), \ldots, w_{m}(t), \alpha(t)\right) \quad t>0 \\
w_{j}(t)=h_{\rho^{j}}\left[y(\cdot) \cdot S_{j} ; w_{j}^{0}\right](t) \quad t>0, \quad \forall j=1, \ldots, m \\
y(0)=x \\
\left(x, w_{1}^{0}, \ldots, w_{m}^{0}\right) \in \mathcal{M},
\end{array}\right.
$$

where $f$ is the controlled dynamics; $y(\cdot) \in \mathbb{R}^{n}$ is the continuous evolution with initial datum $x \in \mathbb{R}^{n} ; w_{j}(\cdot) \in\{-1,1\}$ is the scalar discontinuous output of the delayed thermostat $h_{\rho^{j}}$ applied to the continuous input $y(\cdot) \cdot S_{j}$ where $S_{j}$ is a unit vector of $\mathbb{R}^{n} ; w_{j}^{0} \in\{-1,1\}$ is the initial state of the thermostat; $\mathcal{M} \subset \mathbb{R}^{n} \times \mathbb{R}^{m}$ is a suitable set; and finally $\alpha(\cdot)$ is the measurable control. We also consider the following cost functional

$$
J\left(x, w_{1}^{0}, \ldots, w_{m}^{0}, \alpha\right)=\int_{0}^{+\infty} e^{-\lambda t} \ell\left(y(t), w_{1}(t), \ldots, w_{m}(t), \alpha(t)\right) d t,
$$

where $\ell$ is a suitable running cost, $\lambda>0$ is the discount factor, and $\left(y(\cdot), w_{1}(\cdot), \ldots, w_{m}(\cdot)\right)$ is the evolution in $\mathbb{R}^{n} \times \mathbb{R}^{m}$ given by (1.1). The optimal control problem is then given by the minimization of $J$ over all measurable controls $\alpha$.

A delayed thermostat $h_{\rho}$ (or delayed relay) is, in general, a relationship between a timecontinuous scalar input $u$ and a time-discontinuous scalar output $w \in\{-1,1\}$. It is characterized by the couple of thresholds $\rho=\left(\rho_{1}, \rho_{2}\right)$, with $\rho_{1}<\rho_{2}$, which governs the switching law of the output between 1 and -1 . Such a law presents a particular kind of memory, namely hysteresis. In particular, if $u(t)>\rho_{2}$ (respectively $u(t)<\rho_{1}$ ) then $w(t)=1$ (respectively $w(t)=-1$ ), otherwise, i. e. if $\rho_{1} \leq u(t) \leq \rho_{2}$, the value of $w$ depends on the past

*Dipartimento di Matematica, Università di Trento, Italy, email: bagagiol@science.unitn.it 
evolution of $u$ (see Figure 1). We refer the reader to the next section for more details on the delayed thermostat and to the book by Visintin [20] (and to the references therein), for the general theory of the mathematical models for hysteresis phenomena.

The control problem (1.1), (1.2) can be seen as an optimal control problem for a hybrid evolution $\left(y(\cdot), w_{1}(\cdot), \ldots, w_{m}(\cdot)\right) \in \mathbb{R}^{n} \times\{-1,1\}^{m} \subseteq \mathbb{R}^{n} \times \mathbb{R}^{m}$, where the last $m$ components are the outputs of $m$ thermostats, each one of them respectively subject to the continuous input $y \cdot S_{j}, j=1, \ldots m$. Such switching components may be then interpreted as internal variables of a system whose external observable state is represented by the continuous evolution $y$. In the applications, this kind of hybrid thermostatic evolution naturally arises in several phenomena: whenever an evolution may have more than one modes for evolving, and such modes change from one to another by a sort of internal feedback law. Some examples of such situations are: in biology, for instance when the mode of activity of a colony of bacteria depends on the quantity of nutrient at disposal (see Lenhart-SeidmanYong [17]); in economics, where the mode of production of a good by a firm depends on the cost of production compared with some thresholds depending on the price of the good on the market (see Göcke [16]); again in biology, concerning cross-inhibition networks for the interaction between genes, proteins and other small molecules (see Batt-Belta-Weiss [11]). In [12] Benmansour-Benalia-Djemai-de Leon study the dynamics of a control problem for a so-called "multicellular converter" given by two cells in series, representing some electronic switching circuits. The switching dynamics of that model, consisting on four modes of operation, is very similar to the one considered in the present work. In general, whenever a particular evolution presents multistability, i.e. several (but finite) stable-states, the rules for switching among such states frequently behave as (or can be profitable approximated by) delayed thermostatic rules (see, also for applications to biology, Angeli-Sontag [1], Angeli-Ferrell-Sontag [2]). On the other side, the delayed thermostatic switching rules can be also artificially inserted in the mechanical systems (think of course to the "real" thermostat itself), in order to prevent undesirable behaviors such as fast oscillations in infinitesimal times (the so-called Zeno phenomenon). Moreover, also from an analytical point of view, the artificial insertion of a thermostatic switching rule may be useful. This happens, in optimal control problems for instance, whenever we are facing spatially discontinuous dynamics and/or costs, whose discontinuities are "jumps" through particular surfaces. Such discontinuities may lead to nonexistence of trajectories and (in a dynamic programming framework) to discontinuous Hamilton-Jacobi equations. The insertion of thermostatic approximations of such discontinuities may certainly help in treating such problems (see for instance Liberzon [18] and, for applications to a problem with uncertainty, Bagagiolo-Bauso [7]).

Our goal is to use the dynamic programming technique in order to characterize the value function

$$
V\left(x, w_{1}^{0}, \ldots, w_{m}^{0}\right)=\inf _{\alpha} J\left(x, w_{1}^{0}, \ldots, w_{m}^{0}, \alpha\right)
$$

as the unique viscosity solution of a suitable Hamilton-Jacobi problem. Such a problem consists of a system of Hamilton-Jacobi equations, one for every value of the switching variable $w=\left(w_{1}, \ldots, w_{m}\right) \in\{-1,1\}^{m} \subset \mathbb{R}^{m}$, and defined on a suitable subset $\mathcal{M}_{w} \subset \mathbb{R}^{n}$. These equations are then linked each other by some "implicit" boundary conditions: i.e. boundary conditions which are given by the values of the unknown function itself on other "branches" $\mathcal{M}_{\xi}, \mathcal{M}_{\zeta}$. Using a suitable version of the dynamic programming principle, we first prove that the value function is a continuous viscosity solution on every branch $\mathcal{M}_{w}$ with that "implicit" boundary condition. This is done by recognizing, on every branch, our thermostatic problem as a suitable exit-time problem. Then we construct a suitable operator of contraction type and we prove that every solution of the system is a fixed point of that operator, from which the desired uniqueness follows. However, the boundary data could be discontinuous, since they are given by the glue of the values of the unknown function on 


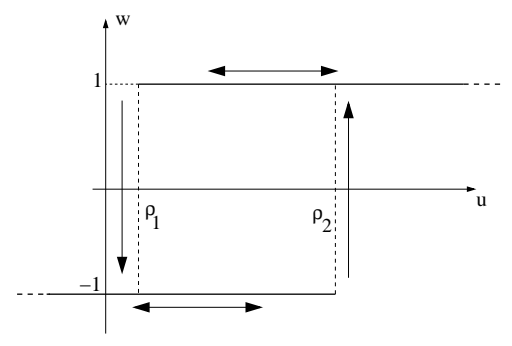

Figure 1: Delayed thermostat with thresholds $\rho=\left(\rho_{1}, \rho_{2}\right)$.

other branches. Hence, in order to implement our method, we have also to slightly adapt some results for exit-time problems to the case of discontinuous exit costs.

We refer the reader to the book by Bardi and Capuzzo Dolcetta [8] for the theory of viscosity solutions and applications to optimal control problems. Other thermostatic problems of this type, but with only one switching variable $w \in\{-1,1\}$, are studied, with similar techniques, for instance in Bagagiolo [4]. In the present case, the presence of more than one thermostatic switching variables leads to some new problems to be treated, such as the discontinuity of the boundary data and the more complex geometry of the branches. These facts also reflect on the construction of the fixed-point operator. For other approaches to hybrid control problems via dynamic programming techniques we refer for instance to Bensoussan-Menaldi [13], Dharmatti-Ramaswamy [15], and Zhang-James [21].

As already said, the presence of more than one switching variables leads to a rather geometrical complexity of the branches $\mathcal{M}_{w} \in \mathbb{R}^{n}$, indeed they are essentially given by the intersection of $m$ half-spaces. This fact also leads to possible hard notations and hence complicated statements. In order to overcome this fact, in this paper we are going to focus our attention to the case where $n=m=2$ (i.e. two-dimensional space-state and two thermostats) and $S_{1}=e_{1}, S_{2}=e_{2}$ (the unit vectors of the canonical basis of $\mathbb{R}^{2}$ ). We refer the reader to Remark 5.2 for comments on a more general case.

The paper is organized in the following way. In Section 2 we say something about the input/output relationship given by the delayed relay (thermostat). In Section 3 we recall something about exit-time problems and in particular we give some new results for a case with a discontinuous exit cost. In Section 4 we say something about the trajectories of an ODE system with thermostatic variables, and give the rigorous formulation of our problem; we address the continuity of the value function, the validity of the Dynamic Programming Principle, and we interpret the value function as the value function of a suitable exit-time problem. In Section 5 we derive the suitable system of Hamilton-Jacobi equations and we prove that it is uniquely solved by the value function in the viscosity sense. In the Appendix we give the proofs of some of the statements in Section 3 and in Section 4.

\section{On the thermostat and thermostatic dynamics}

For more details on this subject we refer to Visintin [20]. Let us consider a continuous input $u:[0,+\infty[\rightarrow \mathbb{R}$, a discontinuous output $w:[0,+\infty[\rightarrow\{-1,1\}$, and two different thresholds for the values of $u$, let us say $\rho_{1}$ and $\rho_{2}$, with $\rho_{1}<\rho_{2}$, for which $w$ respectively switches "down" from +1 to -1 , and "up" from -1 to +1 . We define

$$
\left.\left.\overline{\mathcal{O}}:=(]-\infty, \rho_{2}\right] \times\{-1\}\right) \cup\left(\left[\rho_{1},+\infty[\times\{1\}) \subset \mathbb{R}^{2}=: \overline{\mathcal{O}}_{-1} \cup \overline{\mathcal{O}}_{1},\right.\right.
$$

and we can think to the delayed switching as the evolution of the couple $(u(\cdot), w(\cdot))$ on the set $\overline{\mathcal{O}}$ with a suitable switching rule for switching from one branch to the other (see Figure 
1). More in details, we say that $w$ is the output of the delayed switching rule (or "delayed thermostat", or "delayed relay") with couple of thresholds $\rho=\left(\rho_{1}, \rho_{2}\right)$, input $u$, and initial state $w_{0} \in\{-1,1\}$, and we write $w(t)=h_{\rho}\left[w, w_{0}\right](t) \forall t \geq 0$, if (here $\delta$ is any positive number)

$$
\begin{aligned}
& \text { i) }(u(t), w(t)) \in \overline{\mathcal{O}} \forall t \geq 0, \quad w(0)=w_{0},\left(u(0), w_{0}\right) \in \overline{\mathcal{O}} \\
& \text { ii) } w(t)=1, u(\cdot) \geq \rho_{1} \text { in }[t, t+\delta] \Longrightarrow w(\cdot) \equiv 1 \text { in }[t, t+\delta], \\
& \text { iii) } w(t)=-1, u(\cdot) \leq \rho_{2} \text { in }[t, t+\delta] \Longrightarrow w(\cdot) \equiv-1 \text { in }[t, t+\delta]
\end{aligned}
$$

Such conditions say that $w$ switches if and only if the couple $(u, w)$ is on one of the two switching points $\left(\rho_{1}, 1\right)$ and $\left(\rho_{2},-1\right)$, and the input $u$ crosses the threshold $\rho_{i}, i=1,2$ (decreasing if the threshold is $\rho_{1}$, increasing if it is $\rho_{2}$ ). According to this rule, we call a "switching instant" any instant $t \geq 0$ such that $w(t)=\bar{w} \in\{1,-1\}$, and $w \equiv-\bar{w}$ immediately after $t$ (for instance in $] t, t+\delta]$ ). In particular note that, for instance, the fact that $w(s)=1$ for every $s \in[t, \bar{t}]$ and that $u(\bar{t})=\rho_{1}$, does not implies any switching if the threshold is not crossed (i.e. if there not exists a sequence $t_{n} \rightarrow \bar{t}^{+}$such that $\left.u\left(t_{n}\right)<\rho_{1}\right)$. This in particular means that the switching only occurs "just after" the switching instant.

After any switching instant $t, w$ cannot immediately switch back, because $u$ has to reach the other threshold. This implies the existence of exactly one output, even for fast oscillating inputs, i.e. no Zeno phenomenon may appear.

Remark 2.1 Given a continuous input $u:[0, T] \rightarrow \mathbb{R}$ and a compatible initial state $w_{0}$ (i.e. $\left.\left(u(0), w_{0}\right) \in \overline{\mathcal{O}}\right)$, a possible characterization of the corresponding output $w(\cdot)=h_{\rho}\left[u, w_{0}\right](\cdot)$ is given by the following "variational principle": the output $w$ is the unique measurable function of bounded variation (i.e. $w \in B V(0, T)) w:[0, T] \rightarrow \mathbb{R}$ such that

$$
\begin{aligned}
& \text { i) } w \in X_{\left(u, w_{0}\right)}=\left\{\xi \in B V(0, T) \mid(u(t), \xi(t)) \in \overline{\mathcal{O}} \forall t \in[0, T], \xi(0)=w_{0}\right\}, \\
& \text { ii) } \operatorname{Var}_{[0, t]}(w)=\min _{\xi \in X_{\left(u, w_{0}\right)}} \operatorname{Var}_{[0, t]}(\xi) \forall t \in[0, T]
\end{aligned}
$$

where $\operatorname{Var}_{[0, t]}(\xi)$ means the total variation of $\xi$ in the time interval $[0, t]$. Also note that, for a function $\xi \in X_{\left(u, w_{0}\right)}$ with a finite number of switching in $[0, T]$, as certainly the output $w$ is, such a total variation is exactly given by the number of switchings divided by 2 (the amplitude of the switching). Hence, to minimize the total variation is equivalent to minimize the number of switchings.

Remark 2.2 Referring to exit-time problems (see the next section), we can say that, if at a time $t$ we have, for instance, $(u(t), w(t))=(u(t), 1)$ then certainly $u(t) \in\left[\rho_{1},+\infty[\right.$ and a possible subsequent switching time $\tau \in[t,+\infty[$ is exactly the first exit time of the trajectory $u(\cdot)$ from the closed set $\left[\rho_{1},+\infty[\right.$, and it can be similarly defined as in (3.15).

Hence, our switching rule is linked to the definition of exit from a closed set. Indeed, in the next sections we are going to fit our switching thermostatic control problem in a system of exit-time problems from closed sets. Other possible switching rules may be adopted, for instance the one that refer to the exit-time from the open half lines $]-\infty, \rho_{2}[,] \rho_{1},+\infty[$, which corresponds to the fact that $w$ switches exactly when the input $u$ reaches the threshold. However we prefer the switching rule as exit-time from the closed half lines since the corresponding exit-time control problems, with exit-time from a closed set, are in some sense "more stable" and more suitable to be studied by dynamic programming techniques. See Remark 5.3 for considerations on problems with other thermostatic switching rules.

The following "semigroup property" is often useful.

Proposition 2.3 For every input $u$, initial state $w_{0}$ and instant $\tau \geq 0$ we have 


$$
h_{\rho}\left[u, w_{0}\right](t)=h_{\rho}\left[u(\cdot+\tau), h_{\rho}\left[u, w_{0}\right](\tau)\right](t-\tau) \quad \forall t \geq \tau,
$$

where $u(\cdot+\tau):[0,+\infty[\rightarrow \mathbb{R}$ is the translation $t \mapsto u(t+\tau)$.

\section{Exit-time problems with discontinuous exit costs}

For the general theory and results concerning viscosity solutions for Hamilton-Jacobi equations see Bardi-Capuzzo Dolcetta [8]. Let $\Omega \subset \mathbb{R}^{n}$ be an open set, and $\bar{\Omega}$ its closure. We say that $\Omega$ satisfies a uniform cone property if there exists a constant $c>0$ and a bounded uniformly continuous function $\eta: \bar{\Omega} \rightarrow \mathbb{R}^{n}$ such that

$$
B(x+s \eta(x), c s) \subseteq \Omega \quad \forall x \in \bar{\Omega}, 0<s \leq c,
$$

where $B(x+s \eta(x), c s)$ is the $\mathbb{R}^{n}$-ball centered in $x+s \eta(x)$ with radius $c s$.

Let us now suppose and/or define that

$\Omega \subset \mathbb{R}^{n}$ satisfies the uniform cone property (3.4) :

$F: \mathbb{R}^{n} \times \mathbb{R}^{n} \rightarrow \mathbb{R}$ is a continuous function,

$\xi: \partial \Omega \rightarrow \mathbb{R}$ is a bounded function,

$A \subset \mathbb{R}^{p}$ is compact, $\mathcal{A}=\{\alpha:[0,+\infty[\rightarrow A$, measurable $\}$,

$f: \mathbb{R}^{n} \times \mathcal{A} \rightarrow \mathbb{R}^{n}$ is bounded and Lipschitz continuous,

$\ell: \mathbb{R}^{n} \times \mathcal{A} \rightarrow[0,+\infty[$ is bounded and Lipschitz continuous,

$\lambda>0$,

where, as usual, "Lipschitz continuous" means Lipschitz with respect to $x \in \mathbb{R}^{n}$ uniformly with respect to $a \in A$ (compare with (4.19)).

A continuous function $v: \bar{\Omega} \rightarrow \mathbb{R}$ is said a viscosity solution of the Hamilton-Jacobi equation

$$
\lambda v(x)+F(x, \nabla v(x))=0 \text { in } \Omega,
$$

if, for every $C^{1}$-function $\varphi: \Omega \rightarrow \mathbb{R}$ and for every $x_{0} \in \Omega$ we have the following:

$$
\begin{aligned}
& v-\varphi \text { has a local maximum in } x_{0} \Longrightarrow \lambda v\left(x_{0}\right)+F\left(x_{0}, \nabla \varphi\left(x_{0}\right)\right) \leq 0, \\
& v-\varphi \text { has a local minimum in } x_{0} \Longrightarrow \lambda v\left(x_{0}\right)+F\left(x_{0}, \nabla \varphi\left(x_{0}\right)\right) \geq 0 .
\end{aligned}
$$

A continuous function $v: \bar{\Omega} \rightarrow \mathbb{R}$ is said a viscosity solution of the boundary value problem, with boundary condition in the viscosity sense,

$$
\begin{cases}\lambda v(x)+F(x, \nabla v(x))=0 & \text { in } \Omega \\ v=\xi & \text { on } \partial \Omega\end{cases}
$$

if $v$ is a viscosity solution of the Hamilton-Jacobi equation in $\Omega$ (i.e. the first line) and, for every $x \in \partial \Omega$ and for every $C^{1}$-function $\varphi: \bar{\Omega} \rightarrow \mathbb{R}$ we have

$$
\begin{gathered}
v-\varphi \text { has a local maximum in } x \text { with respect to } \bar{\Omega} \Longrightarrow \\
\min \left(v(x)-\xi_{*}(x), \lambda v(x)+F(x, \nabla \varphi(x))\right) \leq 0 ; \\
v-\varphi \text { has a local minimum in } x \text { with respect to } \bar{\Omega} \Longrightarrow \\
\max \left(v(x)-\xi^{*}(x), \lambda v(x)+F(x, \nabla \varphi(x))\right) \geq 0,
\end{gathered}
$$

where $\xi_{*}$ and $\xi^{*}$ are respectively the lower and upper semicontinuous envelopes:

$$
\xi_{*}(x)=\liminf _{y \rightarrow x, y \in \partial \Omega} \xi(y), \quad \xi^{*}(x)=\limsup _{y \rightarrow x, y \in \partial \Omega} \xi(y), \quad \forall x \in \partial \Omega .
$$


Now we suppose that $\Omega$ and $\xi$ are as in (3.5), but we further assume that there exist $r$ $C^{2}$-functions $\theta_{i}, i=1, \ldots, r$, such that

$$
\Omega=\left\{x \in \mathbb{R}^{n} \mid \theta_{i}(x)>0 \forall i=1, \ldots, r\right\} .
$$

Moreover, we use the following notation

$$
(\partial \Omega)_{i}=\left\{x \in \bar{\Omega} \mid \theta_{i}(x)=0\right\} \subseteq \partial \Omega, \quad \forall i=1, \ldots, r,
$$

and we suppose that every $(\partial \Omega)_{i}$ is a regular $(n-1)$-manifold (possibly with boundary), and that every possible intersection of different $(\partial \Omega)_{i}$ is "transversal" i.e.

$$
x \in(\partial \Omega)_{i_{1}} \cap \cdots \cap(\partial \Omega)_{i_{s}} \Longrightarrow\left\{\nabla \theta_{i_{1}}(x), \ldots, \nabla \theta_{i_{s}}(x)\right\} \text { linearly independent. }
$$

We also suppose that the boundary function $\xi: \partial \Omega \rightarrow \mathbb{R}$ is defined as follows: for every $i=1, \ldots, r$, there exists a continuous and bounded function $\xi_{i}:(\partial \Omega)_{i} \rightarrow \mathbb{R}$, such that $\xi$ satisfies

$$
\xi(x)=\min \left\{\xi_{i}(x) \mid x \in(\partial \Omega)_{i}\right\} \quad \forall x \in \partial \Omega .
$$

Note that (3.12) means that the discontinuities of $\xi$ only occur on "corner points" given by the intersection of some $(\partial \Omega)_{i}$, and that on those points the discontinuity is, in some sense, of "jump type". Moreover, $\xi$ is lower semicontinuous, that is $\xi=\xi_{*}$.

We now consider the controlled dynamical system in $\mathbb{R}^{n}$

$$
\left\{\begin{array}{l}
y^{\prime}(t)=f(y(t), \alpha(t)) \quad t>0 \\
y(0)=x \in \bar{\Omega}
\end{array}\right.
$$

and the payoff (note that, by continuity, $\xi_{*}(x)=\xi^{*}(x)=\xi(x)$ if $x \notin(\partial \Omega)_{i} \cap(\partial \Omega)_{j}$, with $i \neq j)$

$$
J(x, \alpha)=\int_{0}^{t(x, \alpha)} e^{-\lambda t} \ell(y(t), \alpha(t)) d t+e^{-\lambda t(x, \alpha)} \xi_{*}(y(t(x, \alpha))),
$$

where $y(\cdot)$ is the unique trajectory of (3.13) with $\alpha \in \mathcal{A}$, and $t(x, \alpha)$ is the first exit time of the trajectory from $\bar{\Omega}$ :

$$
t(x, \alpha)=\inf \{t \geq 0 \mid y(t) \notin \bar{\Omega}\},
$$

with the convention $\inf \emptyset=+\infty$ (and $\left.e^{-\infty} \xi=0\right)$.

Proposition 3.1 Let (3.5), (3.9), (3.12) hold, let the dynamics be totally controllable on $\partial \Omega$, i.e.:

$$
\begin{aligned}
& \forall x \in \partial \Omega, \exists a_{1}, a_{2} \in A \text { such that } \\
& f\left(x, a_{1}\right) \text { is strictly entering in } \Omega \text { at } x, \\
& f\left(x, a_{2}\right) \text { is strictly entering in the complementary of } \bar{\Omega} \text { at } x,
\end{aligned}
$$

and moreover let us suppose that, for every $i \neq j$, for every $x \in(\partial \Omega)_{i} \cap(\partial \Omega)_{j}$, there exists a neighborhood $U$ of $x$ such that, for every $z \in U \cap(\partial \Omega)_{i}$ there exists a control $\alpha \in A$ whose corresponding trajectory starting from $z$ reaches $(\partial \Omega)_{j}$ in a lap of time $t$ satisfying

$$
t \leq \operatorname{Cdist}\left(z,(\partial \Omega)_{j}\right),
$$

with $C>0$ independent on $z$ and $\alpha$. 
Then, the value function $v$ of the minimization problem

$$
v(x)=\sup _{\alpha \in \mathcal{A}} J(x, \alpha)
$$

is continuous in $\bar{\Omega}$, and it is the unique bounded viscosity solution of the Hamilton-JacobiBellman boundary value problem (with boundary condition in the viscosity sense)

$$
\begin{cases}\lambda v(x)+\max _{a \in A}\{-f(x, a) \cdot \nabla v(x)-\ell(x, a)\}=0 & \text { in } \Omega \\ v=\xi_{*} & \text { on } \partial \Omega\end{cases}
$$

Proof. See the Appendix.

Remark 3.2 Note that condition (3.17) means that, from any point around a "corner" $(\partial \Omega)_{i_{1}} \cap(\partial \Omega)_{i_{2}} \cap \ldots \cap(\partial \Omega)_{i_{s}}$, we can always reach each one of the boundary-branches $(\partial \Omega)_{i_{k}}$, and that we can do it even controlling the time by the initial distance from that boundarybranch.

\section{The optimal bi-thermostatic problem}

In this section we are going to study a bidimensional infinite horizon problem with two thermostatic switchings respectively acting on the "main" directions of $\mathbb{R}^{2}, e^{1}, e^{2}$ (i.e. the unit vectors of the canonical basis of $\mathbb{R}^{2}$ ). Let us consider a compact set of constant controls $A \subset \mathbb{R}^{p}$ and $\mathcal{A}$ the set of measurable control functions

$$
\mathcal{A}=\{\alpha:[0,+\infty[\rightarrow A \mid \alpha \text { is measurable }\}
$$

Let also $f: \mathbb{R}^{2} \times\{-1,1\} \times\{-1,1\} \times A \rightarrow \mathbb{R}^{2}$ and $\ell: \mathbb{R}^{2} \times\{-1,, 1\} \times\{-1,1\} \times A \rightarrow[0,+\infty[$ be two continuous functions such that:

$$
\begin{aligned}
& \exists M>0, \exists L>0, \\
& \text { such that } \forall x, y \in \mathbb{R}^{2}, \forall w, z \in\{-1,1\}, \forall a \in A, \\
& |f(x, w, z, a)| \leq M,|\ell(x, w, z, a)| \leq M, \\
& |f(x, w, z, a)-f(y, w, z, a)| \leq L|x-y|, \\
& |\ell(x, w, z, a)-\ell(y, w, z, a)| \leq L|x-y| .
\end{aligned}
$$

We then consider a discount factor $\lambda>0$; two delayed relays defined as described in Section 2 :

$$
w(t)=h_{\rho}\left[\cdot ; w_{0}\right](t) \text { and } z(t)=h_{\eta}\left[\cdot ; z_{0}\right](t),
$$

where $\rho:=\left(\rho_{1}, \rho_{2}\right)$ and $\eta:=\left(\eta_{1}, \eta_{2}\right)$; and the following controlled thermostatic dynamic system:

$$
\left\{\begin{array}{l}
y^{\prime}(t)=f(y(t), w(t), z(t), \alpha(t)) \quad t>0 \\
w(t)=h_{\rho}\left[y(\cdot) \cdot e_{1} ; w_{0}\right](t) \quad t \geq 0 \\
z(t)=h_{\eta}\left[y(\cdot) \cdot e_{2} ; z_{0}\right](t) \quad t \geq 0 \\
y(0)=x
\end{array}\right.
$$

and the problem of minimizing the following cost functional:

$$
J\left(x, w_{0}, z_{0}, \alpha\right)=\int_{0}^{+\infty} e^{-\lambda s} \ell(y(s), w(s), z(s), \alpha(s)) d s
$$



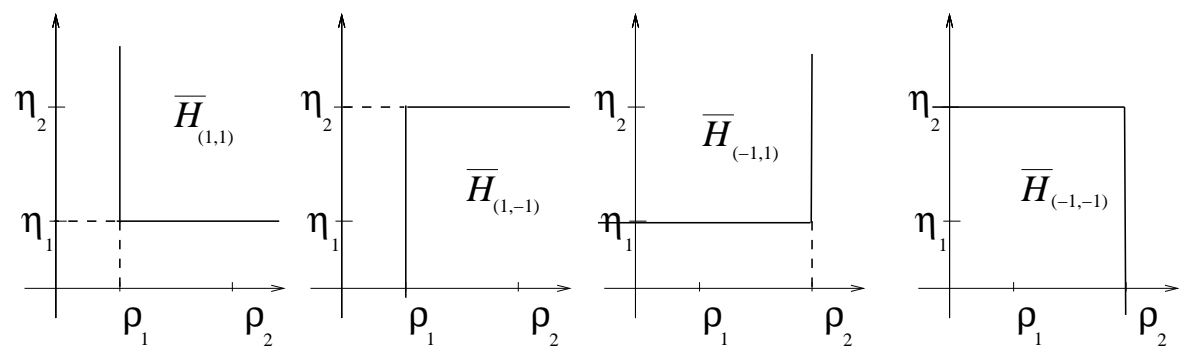

Figure 2: Geometrical representations of the projections of $\overline{\mathcal{H}}_{(w, z)}$, on (the first) $\mathbb{R}^{2}$.

\subsection{The controlled bi-thermostatic system.}

In (4.21) the initial state is the tern $\left(x, w_{0}, z_{0}\right) \in \mathbb{R}^{2} \times\{-1,1\} \times\{-1,1\} \subset \mathbb{R}^{4}$, which is admissible if it satisfies the following conditions:

$$
\begin{aligned}
& w_{0}=1 \quad \text { and } \quad z_{0}=1 \quad \Rightarrow \quad x \cdot e_{1} \geq \rho_{1} \text { and } x \cdot e_{2} \geq \eta_{1} \\
& w_{0}=1 \quad \text { and } \quad z_{0}=-1 \quad \Rightarrow \quad x \cdot e_{1} \geq \rho_{1} \text { and } x \cdot e_{2} \leq \eta_{2} \\
& w_{0}=-1 \quad \text { and } \quad z_{0}=1 \quad \Rightarrow \quad x \cdot e_{1} \leq \rho_{2} \text { and } x \cdot e_{2} \geq \eta_{1} \\
& w_{0}=-1 \quad \text { and } \quad z_{0}=-1 \quad \Rightarrow \quad x \cdot e_{1} \leq \rho_{2} \text { and } x \cdot e_{2} \leq \eta_{2}
\end{aligned}
$$

We define the subset $\overline{\mathcal{H}} \subset \mathbb{R}^{4}$, given by the following four connected components (see Figure 2),

$$
\begin{aligned}
& \overline{\mathcal{H}}:=\overline{\mathcal{H}}_{(1,1)} \cup \overline{\mathcal{H}}_{(1,-1)} \cup \overline{\mathcal{H}}_{(-1,1)} \cup \overline{\mathcal{H}}_{(-1,-1)}, \\
& \overline{\mathcal{H}}_{(1,1)}=\left\{(y, 1,1) \in \mathbb{R}^{4} \mid y \cdot e_{1} \geq \rho_{1}, y \cdot e_{2} \geq \eta_{1}\right\} \\
& \overline{\mathcal{H}}_{(1,-1)}=\left\{(y, 1,-1) \in \mathbb{R}^{4} \mid y \cdot e_{1} \geq \rho_{1}, y \cdot e_{2} \leq \eta_{2}\right\} \\
& \overline{\mathcal{H}}_{(-1,1)}=\left\{(y,-1,1) \in \mathbb{R}^{4} \mid y \cdot e_{1} \leq \rho_{2}, y \cdot e_{2} \geq \eta_{1}\right\} \\
& \overline{\mathcal{H}}_{(-1,-1)}=\left\{(y,-1,-1) \in \mathbb{R}^{4} \mid y \cdot e_{1} \leq \rho_{2}, y \cdot e_{2} \leq \eta_{2}\right\}
\end{aligned}
$$

We then take as admissible initial states for the system $(4.21)$ the states $\left(x, w_{0}, z_{0}\right) \in \overline{\mathcal{H}}$. Hence, in this setting, $\overline{\mathcal{H}}$ is the set $\mathcal{M}$ and the sets $\overline{\mathcal{H}}_{(w, z)}$ are the branches $\mathcal{M}_{w}$, as in the Introduction.

Actually, it is not obvious what a solution of (4.21) is. If we pretend that the thermostatic relationships given by the second and the third lines be satisfied for every time, then the solution may even not exist. Remember that by our definition the switching occurs just after the switching instant, that is when the input has already crossed the threshold: if the input does not cross the threshold, then no switching can occur. Now, if $t^{*}$ is a switching instant, let us say for the first thermostat $h_{\rho}$ from 1 to -1 , this means that $y\left(t^{*}\right) \cdot e_{1}=\rho_{1}$, $y \cdot e_{1} \geq \rho_{1}$ before $t^{*}$ and, just after $t^{*}$, we should have $y \cdot e_{1}<\rho_{1}$. But just after $t^{*}$ the new dynamics is given by $f(y,-1, z, \alpha)$ instead of $f(y, 1, z, \alpha)$ and it may happen that such a new dynamics makes the trajectory $y \cdot e_{1}$ immediately come back, that is not cross the threshold $\rho_{1}$ (whereas, the old dynamics $f(y, 1, z, \alpha)$ makes certainly cross the threshold, otherwise $t^{*}$ cannot be a switching instant). Hence, in such a particular case, if the thermostat does not switch then we must have a switching (since we cross the threshold), on the contrary if the thermostat switches then we cannot have switching (since we do not cross the threshold). The solution definitely does not exist.

However, it is obvious that, in the previous example, $t^{*}$ must be a switching instant for any possible definition of solution $(y(\cdot), w(\cdot), z(\cdot))$ of $(4.21)$. Moreover, it is also obvious that any possible solution of (4.21) must evolve inside the set $\overline{\mathcal{H}}$. Hence, inspired by the variational principle satisfied by the thermostat, see Remark 2.1, we use the following Proposition to define a solution of (4.21). In the sequel, $P C B V_{l o c}(\tau,+\infty)$ means the set of functions on 
$[\tau,+\infty[$ which are piecewise constant and of bounded variation on every compact set. Moreover, for any couple $(w, z) \in P C B V_{l o c}(0,+\infty) \times P C B V_{l o c}(0,+\infty)$, we define $t_{(w, z)}^{0}=0$ and denote by $\left\{t_{(w, z)}^{i}\right\}_{i \in \mathbb{N} \backslash\{0\}}$ the (possibly finite) sequence of the discontinuity instants of $(w, z)$. Finally, for any point $\left(x, w_{0}, z_{0}\right) \in \overline{\mathcal{H}}$, for any control $\alpha \in \mathcal{A}$, and for any instant $\tau \geq 0$, we define the set

$$
\begin{gathered}
X_{\left(x, w_{0}, z_{0}, \alpha, \tau\right)}=\left\{( y , w , z ) \in C ^ { 0 } \left(\left[\tau,+\infty[) \times P C B V_{l o c}(\tau,+\infty) \times P C B V_{l o c}(\tau,+\infty) \mid\right.\right.\right. \\
y(t)=x+\int_{\tau}^{t} f(y(s), w(s), z(s), \alpha(s)) d s \forall t \in[\tau,+\infty[, \\
w(\tau)=w_{0}, z(\tau)=z_{0}, \quad(y(t), w(t), z(t)) \in \overline{\mathcal{H}} \forall t \in[\tau,+\infty[\} .
\end{gathered}
$$

Proposition 4.1 Given an initial state $\left(x, w_{0}, z_{0}\right) \in \overline{\mathcal{H}}$ and a control $\alpha \in \mathcal{A}$, there exists at least one tern $(y, w, z) \in X_{\left(x, w_{0}, z_{0}, \alpha, 0\right)}$ such that, for any $i \in \mathbb{N}$,

$$
t_{(w, z)}^{i+1}-t_{(w, z)}^{i}=\max \left\{t_{(\xi, \zeta)}^{1} \mid(\phi, \xi, \zeta) \in X_{\left(y\left(t_{(w, z)}^{i}\right), w\left(t_{(w, z)}^{i}\right), z\left(t_{(w, z)}^{i}\right), \alpha, t_{(w, z)}^{i}\right)}\right\} .
$$

Proof. Let us note that Proposition 4.1 just says that there exists at least one trajectory $(y, w, z)$ which, whenever it belongs to one of the connected components of $\overline{\mathcal{H}}$, then it remains on it as long as possible (note that $t_{(\xi, \zeta)}^{1}$ is the first switching instant of the trajectory $(\phi, \xi, \zeta)$ which starts at the instant $\tilde{t}=t_{(w, z)}^{i}$ with initial condition $\left.(y(\tilde{t}), w(\tilde{t}), z(\tilde{t}))\right)$. Here we only show how to (almost obviously) construct such a required tern $(y, w, z)$.

We first consider the following system, denoting by $y_{1}$ its unique trajectory defined in $[0,+\infty[$

$$
\left\{\begin{array}{l}
y_{1}^{\prime}(t)=f\left(y_{1}(t), w_{0}, z_{0}, \alpha(t)\right), t>0 \\
y_{1}(0)=x
\end{array}\right.
$$

Let $t_{1} \geq 0$ be the first switching instant of (at least one of) the two thermostats $h_{\rho}\left[y_{1} \cdot e_{1}, w_{0}\right]$, $h_{\eta}\left[y_{1} \cdot e_{2}, z_{0}\right]$ (if they never switch, then the tern $\left(y_{1}, w_{0}, z_{0}\right)$ is the searched one). Let us define $w_{1}=w_{0}$ if $t_{1}$ is not a switching instant for $h_{\rho}\left[y_{1} \cdot e_{1}, w_{0}\right]$ and $w_{1}=-w_{0}$ otherwise. Let us similarly define $z_{1}$. Hence we consider the following system, denoting by $y_{2}$ its unique trajectory in $\left[t_{1},+\infty[\right.$

$$
\left\{\begin{array}{l}
y_{2}^{\prime}(t)=f\left(y_{2}(t), w_{1}, z_{1}, \alpha(t)\right), t>t_{1}, \\
y_{2}\left(t_{1}\right)=y_{1}\left(t_{1}\right) .
\end{array}\right.
$$

Again, let $t_{2} \geq t_{1}$ be the first switching instant for $h_{\rho}\left[y_{2} \cdot e_{1}, w_{1}\right]$ or for $h_{\eta}\left[y_{2} \cdot e_{2}, z_{1}\right]$, and define $w_{2}=w_{1}$ if $t_{2}$ is not a switching instant for $h_{\rho}\left[y_{2} \cdot e_{1}, w_{1}\right]$ and $w_{2}=-w_{1}$ otherwise. Do the same for defining $z_{2}$ and consider the system

$$
\left\{\begin{array}{l}
y_{3}^{\prime}(t)=f\left(y_{3}(t), w_{2}, z_{2}, \alpha(t)\right), t>t_{2}, \\
y_{3}\left(t_{2}\right)=y_{2}\left(t_{2}\right)
\end{array}\right.
$$

We proceed in this way, whenever $t_{i}<+\infty$ and consider the tern $(y, w, z)$ constructed by gluing the pieces $\left(y_{i}, w_{i-1}, z_{i-1}\right)$ each one of them defined in $\left.] t_{i-1}, t_{i}\right]$. We are done if we cover all times $[0,+\infty[$. This is true since the switchings are delayed. Indeed, let us define

$$
\delta=\frac{\min \left\{\rho_{2}-\rho_{1}, \eta_{2}-\eta_{1}\right\}}{M}>0
$$

which represents the minimum necessary time for the trajectory $y$ passing from one threshold to the other of at least one thermostat (here $M$ is a bound for $f(4.19)$ ). Hence, whenever we consider three consecutive instants $t_{i-1}, t_{i}, t_{i+1}$ we have

$$
t_{i+1}-t_{i-1} \geq \delta,
$$

since, after three switchings, at least one of the two thermostats has switched two times. 
Definition 4.2 For any initial state $\left(x, w_{0}, z_{0}\right) \in \overline{\mathcal{H}}$ and for every control $\alpha \in \mathcal{A}$, as solution of (4.21) we mean the function constructed in the proof of Proposition 4.1, and generally we denote it by

$$
(y, w, z)_{\left(x, w_{0}, z_{0}\right)}(\cdot ; \alpha)=\left(y_{\left(x, w_{0}, z_{0}\right)}(\cdot ; \alpha), w_{\left(x, w_{0}, z_{0}\right)}(\cdot ; \alpha), z_{\left(x, w_{0}, z_{0}\right)}(\cdot ; \alpha)\right) .
$$

Remark 4.3 Uniqueness is not guaranteed by Proposition 4.1. Indeed, it does not in general hold. Let $(\tilde{y}, \tilde{w}, \tilde{z})$ be the solution as in Definition 4.2. If $t$ is a switching instant for $(\tilde{y}, \tilde{w}, \tilde{z})$ and if the state $(\tilde{y}(t), \tilde{w}(t), \tilde{z}(t))$ is on the corresponding corner point of $\overline{\mathcal{H}}_{(\tilde{w}(t), \tilde{z}(t))}$, then it may happen that after $t$ we may construct other trajectories than $(\tilde{y}, \tilde{w}, \tilde{z})$ satisfying the maximality requirement. Such a possibility depends on the directions of the four vectors $f(\tilde{y}(t), \tilde{w}(t), \tilde{z}(t)), f(\tilde{y}(t),-\tilde{w}(t),-\tilde{z}(t)), f(\tilde{y}(t), \tilde{w}(t),-\tilde{z}(t))$, and $f(\tilde{y}(t),-\tilde{w}(t), \tilde{z}(t))$ (in particular, on the possible directions of the vectors after switching; see Bagagiolo [3] for some more details). In such particular cases, we may then choose other switchings (i.e other commutations between $w,-w$ and between $z,-z)$ than those chosen for $(\tilde{y}, \tilde{w}, \tilde{z})$.

However, the switchings chosen for $(\tilde{y}, \tilde{w}, \tilde{z})$ are always possible (that is such a maximizing trajectory always exists), whereas other possibilities do not always hold, in general. Moreover, the trajectory $(\tilde{y}, \tilde{w}, \tilde{z})$ chooses the switchings in a sort of non-anticipative way, that is they do not depend on the direction of the field $f$ after the switching. Also, the switchings of $(\tilde{y}, \tilde{w}, \tilde{z}$ ) are in some sense intrinsic (as our real models are: the switching obeys to an internal feedback law): they only depend, via the delayed thermostat, by the evolution on the actual connected component $\overline{\mathcal{H}}_{(\tilde{w}, \tilde{z})}$; whereas other choices seem to more strongly depend on a possible external agent.

However, for our control problem, the possible multiplicity of the solution is not a problem. Indeed we are going to assume some suitable controllability hypotheses on the corner points from which we may approximate any possible other solution by our solution as defined in Definition 4.2.

\subsection{The infinite horizon problem}

For each couple $(w, z) \in\{(1,1),(1,-1),(-1,1),(-1,-1)\}$, we will use the following notations:

i) $\mathcal{H}_{(w, z)}$ for the set defined as in (4.24), but with strict inequalities (i.e. the twodimensional manifold $\overline{\mathcal{H}}_{(w, z)} \subset \mathbb{R}^{4}$ without its boundary);

ii) $\partial \overline{\mathcal{H}}_{(w, z)}$ for the set defined as in (4.24) where at least one equality holds (i.e the boundary of the manifold $\left.\overline{\mathcal{H}}_{(w, z)} \subset \mathbb{R}^{4}\right)$.

iii) $\partial \overline{\mathcal{H}}$ for the set $\bigcup_{w, z=1,-1} \partial \overline{\mathcal{H}}_{(w, z)}$.

iv) $\partial_{e_{i}} \mathcal{H}_{(w, z)}$ (respectively $\left.\bar{\partial}_{e_{i}} \mathcal{H}_{(w, z)}\right) i=1,2$ for the open (respectively closed) half line along the direction $e_{i}$ contained in the boundary of $\overline{\mathcal{H}}_{(w, z)}$ (i.e. the part of the boundary which is parallel to $e_{i}$ without or with the corner point).

In Figure 2, the four connected components of $\overline{\mathcal{H}}$ are represented. Each one is composed by an "interior space", $\mathcal{H}_{(w, z)}$ and two boundary half lines (each one along the two directions of $\left.\mathbb{R}^{2}\right), \bar{\partial}_{e_{1}} \mathcal{H}_{(w, z)}$ and $\bar{\partial}_{e_{2}} \mathcal{H}_{(w, z)}$, which have in common only one "corner point". We denote by $\sigma_{(w, z)}$ such a corner point, for every $\overline{\mathcal{H}}_{(w, z)}$ :

$$
\begin{aligned}
& \sigma_{(1,1)}=\left(\rho_{1}, \eta_{1}, 1,1\right), \sigma_{(1,-1)}=\left(\rho_{1}, \eta_{2}, 1,-1\right), \\
& \sigma_{(-1,1)}=\left(\rho_{2}, \eta_{1},-1,1\right), \sigma_{(-1,-1)}=\left(\rho_{2}, \eta_{2},-1,-1\right) .
\end{aligned}
$$

The value function of the infinite horizon problem is defined as

$$
V(x, w, z):=\inf _{\alpha \in \mathcal{A}} J(x, w, z, \alpha) \quad \forall(x, w, z) \in \overline{\mathcal{H}} .
$$


We suppose the following controllability properties hold on the switching boundaries:

$$
\begin{aligned}
& \forall(x, w, z) \in \partial_{e_{2}} \mathcal{H}_{(w, z)} \exists a_{1}, a_{2} \in A \text { such that: } \\
& \quad f\left(x, w, z, a_{1}\right) \cdot e_{1}<-c<0<c<f\left(x, w, z, a_{2}\right) \cdot e_{1}, \\
& \forall(x, w, z) \in \partial_{e_{1}} \mathcal{H}_{(w, z)} \exists a_{3}, a_{4} \in A \text { such that : } \\
& \quad f\left(x, w, z, a_{3}\right) \cdot e_{2}<-c<0<c<f\left(x, w, z, a_{4}\right) \cdot e_{2} .
\end{aligned}
$$

where $c>0$ is independent from $(x, w, z)$. Condition (4.26) means that, on the points of the boundary (i.e. on the switching points) we can always cross the boundary as well as do not cross it (i.e. we can always make the corresponding variable switch as well as not switch).

We also consider the following hypotheses on the four corner points:

$$
\begin{aligned}
& \forall(w, z) \exists a, a_{1}, a_{2} \in A \text { such that } \\
& w f\left(\sigma_{(w, z)}, a\right) \cdot e_{1}>0 \text { and } z f\left(\sigma_{(w, z)}, a\right) \cdot e_{2}>0, \\
& w f\left(\sigma_{(w, z)}, a_{1}\right) \cdot e_{1}<0<z f\left(\sigma_{(w, z)}, a_{1}\right) \cdot e_{2}, \\
& z f\left(\sigma_{(w, z)}, a_{2}\right) \cdot e_{2}<0<w f\left(\sigma_{(w, z)}, a_{2}\right) \cdot e_{2} .
\end{aligned}
$$

Note that (4.27) means that on every corner point we have at disposal a direction which permits to remain inside the branch $\overline{\mathcal{H}}_{(w, z)}$ (i.e. to not cross any threshold), and two directions, one per threshold, which make cross only one threshold. Also, the second two lines of (4.27) imply:

$$
\begin{aligned}
& \forall(w, z) \exists C>0 \text { and } U \text { neighborhood of } \sigma_{(w, z)} \text { such that } \\
& \forall i, j=1,2,, i \neq j, \forall(x, w, z) \in \partial_{e_{i}} \mathcal{H}_{(w, z)} \cap U \\
& \exists \alpha \in \mathcal{A}, \exists t \leq C \operatorname{dist}\left((x, w, z), \partial_{e_{j}} \mathcal{H}_{(w, z)}\right) \\
& \text { such that }(y, w, z)_{(x, w, z)}(t ; \alpha) \in \partial_{e_{j}} \mathcal{H}_{(w, z)} .
\end{aligned}
$$

Note that (4.28) means that, around any corner, starting from any border point in a suitable neighborhood, we can reach points in the other boundary line with a lap of time infinitesimal as the distance from that portion of boundary (compare with (3.17)).

\subsection{Continuity and DPP}

We always assume the standard hypotheses on the dynamics $f$ and on the cost $\ell$, (4.19), and the controllability hypotheses (4.26), (4.27). For the proof of the following proposition see the Appendix.

Proposition 4.4 The value function $V$ is bounded and continuous in $\overline{\mathcal{H}}$.

Proposition 4.5 The Dynamic Programming Principle (DPP) holds:

$$
\begin{aligned}
& V(x, w, z)=\inf _{\alpha \in \mathcal{A}}\left\{\int_{0}^{t} e^{-\lambda s} \ell\left(y_{\left(x, w_{0}, z_{0}\right)}(s ; \alpha), w_{\left(x, w_{0}, z_{0}\right)}(s ; \alpha), \alpha(s)\right) d s\right. \\
& \left.e^{-\lambda t} V\left(y_{\left(x, w_{0}, z_{0}\right)}(t ; \alpha), w_{\left(x, w_{0}, z_{0}\right)}(t ; \alpha), z_{\left(x, w_{0}, z_{0}\right)}(t ; \alpha)\right)\right\}, \quad \forall t \geq 0, \forall(x, w, z) \in \overline{\mathcal{H}}_{(w, z)} .
\end{aligned}
$$

Proof. The proof is almost standard (see Bardi-Capuzzo Dolcetta [8]) and follows from usual properties of trajectories and the semigroup property of the delayed thermostat, Proposition 2.3 .

\subsection{The exit time problem}

Let the hypotheses on $f$ and $\ell$, (4.19), and the controllability ones (4.26), (4.27) hold. Our aim is to describe the bi-thermostatic optimal control problem as a family of exit-time 
problems, suitably coupled. Given an initial state $\left(x, w_{0}, z_{0}\right) \in \overline{\mathcal{H}}$, and a control $\alpha \in \mathcal{A}$, we define the first switching time as

$$
t_{\left(x, w_{0}, z_{0}\right)}(\alpha)=\inf \left\{t \geq 0 \mid(w, z)_{\left(x, w_{0}, z_{0}\right)}(t ; \alpha) \neq\left(w_{0}, z_{0}\right)\right\},
$$

where we convey $\inf \emptyset=+\infty$. Note that the first switching time is, in other words, the first exit-time from the initial closed connected component $\overline{\mathcal{H}}_{\left(w_{0}, z_{0}\right)}$, and also that, by the swithcing definition of the delayed thermostat, we have $(w, z)_{\left(x, w_{0}, z_{0}\right)}\left(t_{\left(x, w_{0}, z_{0}\right)}(\alpha) ; \alpha\right)=\left(w_{0}, z_{0}\right)$ and $(w, z)_{\left(x, w_{0}, z_{0}\right)}(t+\delta ; \alpha) \neq\left(w_{0}, z_{0}\right)$ for any sufficiently small $\delta>0$.

Now, for any bounded and continuous function $u: \overline{\mathcal{H}} \rightarrow \mathbb{R}$, we define the function $\psi[u]: \partial \overline{\mathcal{H}} \rightarrow \mathbb{R}:$

$$
\psi[u](x, w, z)= \begin{cases}u(x, w,-z) & \text { if }(x, w, z) \in \partial_{e_{1}} \mathcal{H}_{(w, z)}, \\ u(x,-w, z) & \text { if }(x, w, z) \in \partial_{e_{2}} \mathcal{H}_{(w, z)}, \\ \min \{u(x,-w, z), u(x, w,-z)\} & \text { if }(x, w, z)=\sigma_{(w, z)} .\end{cases}
$$

Note that, on a single branch-boundary $\partial \overline{\mathcal{H}}_{(w, z)}$, the value of $\psi[u]$ on a point $(x, w, z) \neq \sigma_{(w, z)}$ is exactly the value of $u$ in a suitable point of another branch, which is nothing but the point where a possible trajectory switches on, when it exits from $\overline{\mathcal{H}}_{(w, z)}$ through $(x, w, z)$. If instead $(x, w, z)$ is the corner point $\sigma_{(w, z)}$, then $\psi[u]\left(\sigma_{(w, z)}\right)$ is the minimum of the two values given by $u$ evaluated on the two points where a possible trajectory may respectively switch on, when it exits from $\overline{\mathcal{H}}_{(w, z)}$ through $\sigma_{(w, z)}$ making one threshold only switch.

By the continuity of the function $u$ and by the definition of $\psi[u]$ on the corner points $\sigma_{(w, z)}$, we immediately get the continuity of $\psi[u]$ on the sets $\partial_{e_{i}} \mathcal{H}_{(w, z)}$ and its lower semicontinuity on $\partial \overline{\mathcal{H}}$ (i.e. the "gluing" of $\psi[u]$ on the corner points is "lower semicontinuous").

Proposition 4.6 For every fixed couple $(w, z)=( \pm 1, \pm 1)$, the restriction of $V$ to the connected component $\overline{\mathcal{H}}_{(w, z)}$, i.e. the function

$$
\left\{x \in \mathbb{R}^{2} \mid(x, w, z) \in \overline{\mathcal{H}}_{(w, z)}\right\} \ni x \mapsto V(x, w, z),
$$

coincides with the value function of the exit-time problem from the closed set $\overline{\mathcal{H}}_{(w, z)}$, with exit-cost given by $\psi[V](\cdot, w, z)$; which is equivalent to say that

$$
\begin{aligned}
& V(x, w, z)=\inf _{\alpha \in \mathcal{A}}\left\{\int_{0}^{t_{(x, w, z)}(\alpha)} e^{-\lambda t} \ell\left(y_{(x, w, z)}(t ; \alpha), w, z\right) d t\right. \\
& \left.+e^{-\lambda t_{(x, w, z)}(\alpha)} \psi[V]\left(y_{(x, w, z)}\left(t_{(x, w, z)}(\alpha) ; \alpha\right), w, z\right)\right\} .
\end{aligned}
$$

Proof. We are going to directly prove (4.30). Let us fix $(x, w, z) \in \overline{\mathcal{H}}$ and $\alpha \in \mathcal{A}$, and denote $\bar{t}=t_{(x, w, z)}(\alpha)$. Hence, by the Dynamic Programming Principle, we have

$$
V(x, w, z) \leq \int_{0}^{\bar{t}} e^{-\lambda t} \ell\left(y_{(x, w, z)}(t ; \alpha), w, z\right) d t+e^{-\lambda \bar{t}} V\left(y_{(x, w, z)}(\bar{t} ; \alpha), w, z\right) .
$$

By the controllability hypotheses, on $(\bar{\xi}, w, z)=\left(y_{(x, w, z)}(\bar{t} ; \alpha), w, z\right) \in \partial \overline{\mathcal{H}}_{(w, z)}$, we have

$$
\begin{array}{ll}
V(\bar{\xi}, w, z) \leq V(\bar{\xi},-w, z) & \text { if }(\bar{\xi}, w, z) \in \partial_{e_{2}} \mathcal{H}_{(w, z)}, \\
V(\bar{\xi}, w, z) \leq V(\bar{\xi}, w,-z) & \text { if } \left.(\bar{\xi}, w, z) \in \partial_{e_{1}} \mathcal{H}_{(w, z)}\right) \\
V(\xi, w, z) \leq \min \{V(\bar{\xi},-w, z), V(\bar{\xi}, w,-z), V(\bar{\xi},-w,-z)\} & \text { if }(\bar{\xi}, w, z)=\sigma_{(w, z)}
\end{array}
$$


The inequalities in the first and in the second lines of (4.32) come from the fact that if we start from a switching point $(\bar{\xi}, w, z)$, then we have more trajectories at our disposal than the ones from the point we "drop on" after the switching: we have all the trajectories starting from the latter point (by controllability) and also all the possible non-switching trajectories starting from $(\bar{\xi}, w, z)$. The inequality in the third line comes again by the controllability on the corner points (4.27), noting that, all the trajectories starting from $(\bar{\xi},-w,-z)$ are easily approximated by trajectories starting from anyone of the three points $(\bar{\xi}, w, z),(\bar{\xi},-w, z),(\bar{\xi}, w,-z)$. Similarly, on the corner points we have

$$
\min \{V(\bar{\xi},-w, z), V(\bar{\xi}, w,-z), V(\bar{\xi},-w,-z)\}=\min \{V(\bar{\xi},-w, z), V(\bar{\xi}, w,-z)\} .
$$

By (4.32) we then definitely conclude that

$$
V(x, w, z) \leq \psi[V](x, w, z) \quad \forall(x, w, z) \in \mathcal{H}_{(w, z)} .
$$

By (4.31) and (4.33), we then get

$$
V(x, w, z) \leq \int_{0}^{\bar{t}} e^{-\lambda t} \ell\left(y_{(x, w, z)}(t ; \alpha), w, z\right) d t+e^{-\lambda \bar{t}} \psi[V](\bar{\xi}, w, z),
$$

from which, by the arbitrariness of $\alpha \in \mathcal{A}$ we get the " $\leq$ " inequality in (4.30).

To obtain the reverse inequality, let us fix $\varepsilon>0$ and take $\alpha_{\varepsilon} \in \mathcal{A}$ such that $J(x, w, z)-\varepsilon \leq$ $V(x, w, z)$, and denote by $\left(y_{\varepsilon}, w_{\varepsilon}, z_{\varepsilon}\right)$ the corresponding trajectory starting from $(x, w, z)$. Again, we also denote $\bar{t}=t_{(x, w, z)}\left(\alpha_{\varepsilon}\right)$, and suppose that $\bar{t}<+\infty$ (which is not restrictive). Moreover, let us take $\delta>0$ such that the trajectory does not switch again in the time interval ] $\bar{t}, \bar{t}+\delta]$. We get

$$
\begin{aligned}
& V(x, w, z) \geq \int_{0}^{+\infty} e^{-\lambda t} \ell\left(y_{\varepsilon}(t), w_{\varepsilon}(t), z_{\varepsilon}(t), \alpha_{\varepsilon}(t)\right) d t-\varepsilon \\
& =\int_{0}^{\bar{t}+\delta} e^{-\lambda t} \ell\left(y_{\varepsilon}(t), w_{\varepsilon}(t), z_{\varepsilon}(t), \alpha_{\varepsilon}(t)\right) d t+\int_{\bar{t}+\delta}^{+\infty} e^{-\lambda t} \ell\left(y_{\varepsilon}(t), w_{\varepsilon}(t), z_{\varepsilon}(t), \alpha_{\varepsilon}(t)\right) d t-\varepsilon \\
& \geq \int_{0}^{\bar{t}+\delta} e^{-\lambda t} \ell\left(y_{\varepsilon}(t), w_{\varepsilon}(t), z_{\varepsilon}(t), \alpha_{\varepsilon}(t)\right) d t+e^{-\lambda(\bar{t}+\delta)} V\left(y_{\varepsilon}(\bar{t}+\delta), w_{\varepsilon}(\bar{t}+\delta), z_{\varepsilon}(\bar{t}+\delta)\right)-\varepsilon .
\end{aligned}
$$

By the continuity of $V$, and by (4.32), letting $\delta \rightarrow 0^{+}$, we then get

$$
V(x, w, z) \geq \int_{0}^{\bar{t}} e^{-\lambda t} \ell\left(y_{\varepsilon}(t), w_{\varepsilon}(t), z_{\varepsilon}(t), \alpha_{\varepsilon}(t)\right) d t+e^{-\lambda \bar{t}} \psi[V]\left(y_{\varepsilon}(\bar{t}), w, z\right)-\varepsilon .
$$

By the arbitrariness of $\varepsilon>0$ we finally get the conclusion.

\section{The Hamilton-Jacobi system}

For an unknown function $u: \overline{\mathcal{H}} \rightarrow \mathbb{R}$, we consider the following Hamilton-Jacobi problem

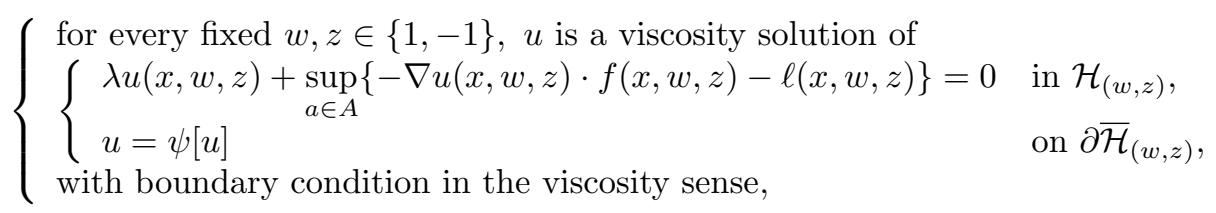

where the symbol " $\nabla$ " means the gradient with respect to the spatial variable only, $x \in \mathbb{R}^{n}$. Let us note that every $\mathcal{H}_{(w, z)}$ is defined as $\Omega$ in (3.9) (with two boundary-branches $\bar{\partial}_{e_{i}} \mathcal{H}_{(w, z)}$, $i=1,2)$, and that, when $u$ is continuous, $\psi[u]$ plays the same role as $\xi_{*}$ with $\xi$ as in (3.12). 
By Proposition 4.6, and by Proposition 3.1, we immediately get that the value function $V$ solves (5.34). Moreover note that Proposition 3.1 also speaks about uniqueness. In the present setting, this means that, once we know $V$ and have calculated $\psi[V]$, then $V$ is indeed the unique solution of every sub-problems on $\overline{\mathcal{H}}_{(w, z)}$, with boundary condition given by $\psi[V]$ itself. However, this (sub-) uniqueness, does not immediately imply the uniqueness of $V$ as solution of (5.34), since in the whole problem on $\overline{\mathcal{H}}$, the boundary conditions are of course intrinsically given through the solution. Uniqueness is the subject of the following proposition.

Proposition 5.1 The value function $V$ is the unique bounded and continuous function from $\overline{\mathcal{H}}$ to $\mathbb{R}$ which solves problem (5.34).

Proof. For every function $v: \overline{\mathcal{H}} \rightarrow \mathbb{R}$, and for every fixed $w, z \in\{1,-1\}$, we will denote by $H J_{(w, z)}[v]$ the corresponding (sub-) Hamilton-Jacobi problem

$$
\begin{cases}\lambda u(x, w, z)+\sup _{a \in A}\{-\nabla u(x, w, z) \cdot f(x, w, z)-\ell(x, w, z)\}=0 & \text { in } \mathcal{H}_{(w, z)}, \\ u=\psi[v] & \text { on } \partial \overline{\mathcal{H}}_{(w, z)},\end{cases}
$$

where $u: \overline{\mathcal{H}}_{(w, z)} \rightarrow \mathbb{R}$ is the unknown function. Moreover, let us denote by $X$ the space of bounded and continuous functions from $\overline{\mathcal{H}}$ to $\mathbb{R}$. This is a complete space when endowed by the sup-norm

$$
\|u\|=\sup _{(x, w, z) \in \overline{\mathcal{H}}}|u(x, w, z)| \quad \forall u \in X .
$$

Our aim is to construct an operator $T: X \rightarrow X$ which has a unique fixed point (i.e. $T[u]=u$ ), and such that any solutions of (5.34) which belongs to $X$ is a fixed point of $T$. This will conclude the proof. Given a function $u \in X$, then $T[u] \in X$ is constructed as follows: for every $w, z \in\{1,-1\}$, the restriction of $T[u]$ to the component $\overline{\mathcal{H}}_{(w, z)}$ is the unique bounded and continuous solution of $H J_{(w, z)}[u]$. By Proposition 3.1, we know that, on every component $\overline{\mathcal{H}}_{(w, z)}, T[u]$ coincides with the value function of the exit-time problem from $\overline{\mathcal{H}}_{(w, z)}$ with exit-cost given by $\psi[u]$, and with dynamics, running cost and discount factor given by $f, \ell$ and $\lambda$ respectively. In other words, for every fixed $w, z \in\{1,-1\}$, and for every $x \in \mathbb{R}^{n}$ such that $(x, w, z) \in \overline{\mathcal{H}}_{(w, z)}$,

$$
\begin{aligned}
& T[u](x, w, z)=\inf _{\alpha \in \mathcal{A}}\left(\int_{0}^{t_{(x, w, z)}(\alpha)} e^{-\lambda t} \ell\left(y_{(x, w, z)}(t ; \alpha), w, z\right) d t\right. \\
& \left.+e^{-\lambda t_{(x, w, z)}(\alpha)} \psi[u]\left(y_{(x, w, z)}\left(t_{(x, w, z)}(\alpha) ; \alpha\right), w, z\right)\right) .
\end{aligned}
$$

It is obvious that any bounded and continuous viscosity solution of (5.34) is a fixed point of $T$. Moreover, we already know that $V$ solves (5.34) and hence it is a fixed point (a fixed point exists). To prove that $T$ has a unique fixed point we prove that $T^{3}=T \circ T \circ T$ is a contraction on $X$.

To simplify notations, let us consider a point $(x, 1,1) \in \overline{\mathcal{H}}_{(1,1)}$. Let us take $u, v \in X$, $\varepsilon>0$ and a control $\alpha_{1} \in \mathcal{A}$ which is $\varepsilon$-optimal for $T^{3}[v](x, 1,1)$ (i.e. for the exit-time problem from $\overline{\mathcal{H}}_{(1,1)}$, with initial point $(x, 1,1)$ and exit-cost $\left.\psi\left[T^{2}[v]\right]\right)$. Moreover, let us denote by $y_{1}$ the corresponding trajectory in $\mathbb{R}^{n}$, and by $t_{1}$ the first exit-time $t_{(x, 1,1)}\left(\alpha_{1}\right)$. Then, by (5.35), we have

$$
\left(T^{3}[u]-T^{3}[v]\right)(x, 1,1)=e^{-\lambda t_{1}}\left(\psi\left[T^{2}[u]\right]\left(y_{1}\left(t_{1}\right), 1,1\right)-\psi\left[T^{2}[v]\right]\left(y_{1}\left(t_{1}\right), 1,1\right)\right)+\varepsilon .
$$

Note that $\left(y_{1}\left(t_{1}\right), 1,1\right)$ is a point of the boundary $\partial \overline{\mathcal{H}}_{(1,1)}$ and hence 

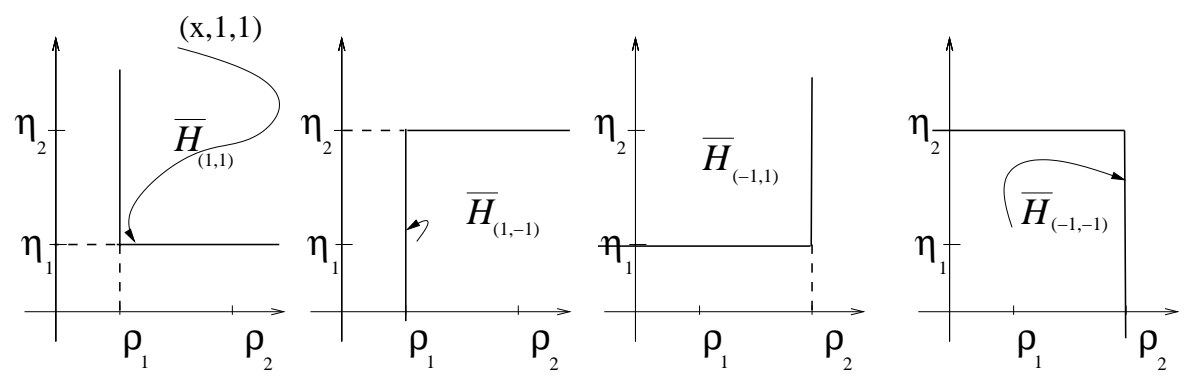

Figure 3: Graphic explanation for part of the proof of Proposition 5.1

$$
y_{1}\left(t_{1}\right) \cdot S_{1}=\rho_{1} \quad \text { or } \quad y_{1}\left(t_{1}\right) \cdot S_{2}=\eta_{1} .
$$

Now, by definition of $\psi$, supposing for instance $\psi\left[T^{2}[v]\right]\left(y_{1}\left(t_{1}\right), 1,1\right)=T^{2}[v]\left(y_{1}\left(t_{1}\right), 1,-1\right)$, we obtain

$$
\left(T^{3}[u]-T^{3}[v]\right)(x, 1,1) \leq e^{-\lambda t_{1}}\left(T^{2}[u]\left(y_{1}\left(t_{1}\right), 1,-1\right)-T^{2}[v]\left(y_{1}\left(t_{1}\right), 1,-1\right)\right)+\varepsilon .
$$

Repeating such an argument, we take $\alpha_{2} \in \mathcal{A}$ E-optimal for $T^{2}[v]\left(y_{1}\left(t_{1}\right), 1,-1\right)$ (as exittime problem from $\overline{\mathcal{H}}_{(1,-1)}$, starting from $\left(y_{1}\left(t_{1}\right), 1,-1\right)$, with exit-cost given by $\left.\psi[T[v]]\right)$, we correspondingly define $y_{2}$ and $t_{2}$, and suppose for instance that $\psi[T[v]]\left(y_{2}\left(t_{2}\right), 1,-1\right)=$ $T[v]\left(y_{2}\left(t_{2}\right),-1,-1\right)$. We then get

$$
\left(T^{3}[u]-T^{3}[v]\right)(x, 1,1) \leq e^{-\lambda\left(t_{1}+t_{2}\right)}\left(T[u]\left(y_{2}\left(t_{2}\right),-1,-1\right)-T[v]\left(y_{2}\left(t_{2}\right),-1,-1\right)\right)+2 \varepsilon .
$$

Also in this case let us note that $\left(y_{2}\left(t_{2}\right), 1,-1\right)$ is a point of the boundary $\partial \overline{\mathcal{H}}_{(1,-1)}$ and hence

$$
y_{2}\left(t_{2}\right) \cdot S_{1}=\rho_{1} \text { or } y_{2}\left(t_{2}\right) \cdot S_{2}=\eta_{2} .
$$

Again, with obvious notations, and supposing $\psi[v]\left(y_{3}\left(t_{3}\right),-1,-1\right)=v\left(y_{3}\left(t_{3}\right), 1,-1\right)$, we finally have

$$
\begin{aligned}
& \left(T^{3}[u]-T^{3}[v]\right)(x, 1,1) \leq e^{-\lambda\left(t_{1}+t_{2}+t_{3}\right)}\left(u\left(y_{3}\left(t_{3}\right), 1,-1\right)-v\left(y_{3}\left(t_{3}\right), 1,-1\right)\right)+3 \varepsilon \\
& \leq e^{-\lambda\left(t_{1}+t_{2}+t_{3}\right)}\|u-v\|+3 \varepsilon
\end{aligned}
$$

noting again that $\left(y_{3}\left(t_{3}\right),-1,-1\right)$ is a point of the boundary $\partial \overline{\mathcal{H}}_{(-1,-1)}$ and hence

$$
y_{3}\left(t_{3}\right) \cdot S_{1}=\rho_{2} \quad \text { or } \quad y_{3}\left(t_{3}\right) \cdot S_{2}=\eta_{2} .
$$

In Figure 3 a trajectory starts from $(x, 1,1)$ and evolves, with dynamcis $f(\cdot, 1,1)$, in $\overline{\mathcal{H}}_{(1,1)}$ until the time $t_{1}$, at which it exits trhough a point $\left(y_{1}\left(t_{1}\right), 1,1\right)$ whose second coordinate is $\eta_{1}$. Then it restarts to move, with dynamics $f(\cdot, 1,-1)$, inside $\overline{\mathcal{H}}_{(1,-1)}$ from the point $\left(y_{1}\left(t_{1}\right), 1,-1\right)$ and, after a time $t_{2}$ it exits through a point $\left(y_{2}\left(t_{2}\right), 1,-1\right)$ whose first coordinate is $\rho_{1}$. After that, it moves inside $\overline{\mathcal{H}}_{(-1,-1)}$, with dynamics $f(\cdot,-1,-1)$, starting from the point $\left(y_{2}\left(t_{2}\right),-1,1\right)$ and, after a time $t_{3}$ it exits through a point $\left(y_{3}\left(t_{3}\right),-1,-1\right)$ whose second coordinate is $\rho_{2}$.

Now, recalling (5.36), (5.37) and (5.39), we can note that in the lap of time $t_{1}+t_{2}+t_{3}$ the trajectory given by the glue of $\left(y_{1}(\cdot), 1,1\right),\left(y_{2}(\cdot), 1,-1\right)$ and $\left(y_{3}(\cdot),-1,-1\right)$ passes three times through some points of the boundaries (the exit points) which also correspond to some 
thresholds points of the thermostats. Since the thermostats are two, this implies that both thresholds of at least one thermostat are reached by the trajectories. By (4.25), this implies that

$$
t_{1}+t_{2}+t_{3} \geq \delta>0
$$

where $\delta$ is independent from the starting point $(x, 1,1)$ and from the $\varepsilon$-optimal controls $\alpha_{1}, \alpha_{2}, \alpha_{3}$, but only depends on the dynamics $f$ and on the thresholds of the thermostats. Hence, from (5.38), by the arbitrariness of $(x, 1,1)$ and of $\varepsilon>0$, we get

$$
\left\|T^{3}[u]-T^{3}[v]\right\| \leq e^{-\lambda \delta}\|u-v\| \quad \forall u, v \in X,
$$

where, since $\lambda>0,0<e^{-\lambda \delta}<1$, and the proof is completed.

Remark 5.2 The two-dimensional model we have studied here may be seen as the prototype for the $n$-dimensional case of $m$ thermostats, with $m \leq n$ and $S_{1}, \ldots, S_{m}$ linearly independent.

A more general situation which can be studied with the same argumentation as here, but obviously with a strongly more complicated geometrical description, is the case where $m$ is arbitrary and the unit vectors $S_{j}$ are pair-wise non parallel (even if they may be linearly dependent). Indeed, in such a case, every branch $\mathcal{M}_{w}$ (using the notations as in the Introduction) is not empty, from every branch it is possible to switch on some other branches, and every branch can be reached by a suitable switching from some other branches.

Otherwise, if some $S_{i}$ and $S_{j}$ with $i \neq j$ are parallel, then some branches may be empty and also some non empty branches may be unreachable, that is it is impossible to switch on it, but it is only possible to switch out from it. Hence it could happen that some internal switching loops appear, which involve only a restricted number of branches. In such a case the model is slightly different from the one here studied. A simpler situation like that, only for a one-dimensional model, was studied in Bagagiolo [3]. However, such a situation (which roughly speaking means: different thermostats applied to the same component) is probably better suitable to approximate more general hysteresis-memory laws (as the Preisach one) than to be applied to our switching model problems as described in the Introduction.

Remark 5.3 In this work we have assumed that the "switching thermostatic rule" is linked to the "exit-rule" from a closed set (i.e. the switching occurs "just after" the switching time). As said in Remark 2.2, other switching rules may be assumed. Let us consider the problem where thermostats switch "exactly when" the threshold is reached. This corresponds to a problem with state-space

$$
\mathcal{H}=\bigcup_{(w, z)} \mathcal{H}_{(w, z)}
$$

which may be interpreted as the "interior" of $\overline{\mathcal{H}} \subset \mathbb{R}^{4}$. Let $\tilde{V}$ be the value function of the problem (with the same $f, \ell, \lambda$ as before) with such a kind of switching rule. Then, by the controllability hypotheses, we certainly have $\tilde{V}=V$ on $\mathcal{H}$, where $V$ is the value function as in the previous part of this work. Indeed, the inequality $V \leq \tilde{V}$ is almost immediate, since any trajectory for $\tilde{V}$ may be easily approximated by a trajectory for $\tilde{V}$. On the other hand, the inequality $\tilde{V} \leq V$ can be obtained by suitably using the estimates (6.40)-(6.42), possibly restricting the branch $\mathcal{H}_{(w, z)}$ by cutting a small $\varepsilon$-strip around the boundary $\partial \overline{\mathcal{H}}_{(w, z)}$ (remember that the trajectories for $V$ may run along the boundary $\partial \overline{\mathcal{H}}$, whereas the trajectories for $\tilde{V}$ may not (the boundary is not admissible for that problem)).

In the same way, other possible "mixed" switching rules lead to value functions which coincide with $V$ on the common sets of definition.

Of course, only $V$ is the good one to be searched as solution of the Hamilton-Jacobi problem, since it is the unique that has all the boundary values. 


\section{Appendix}

Proof of Proposition 3.1. $v$ is continuous. The continuity of $v$ comes from (3.16), (3.17), from the fact that $\xi$ is separately continuous on every $(\partial \Omega)_{i}$, and also from the fact that $\xi_{*}$ is continuous on $(\partial \Omega)_{1} \cap(\partial \Omega)_{\tilde{1}}$ (see (3.12)). In particular note that (3.17) guarantees the (controlled in time) reachability of $(\partial \Omega)_{1}$, where the values of the exit cost $\xi$ are (probably) lower than the values on $(\partial \Omega)_{\tilde{1}}$, at least for points near to $(\partial \Omega)_{1} \cap(\partial \Omega)_{\tilde{1}}$. Indeed, about continuity, adapting a result due to Soner [19] (see also Bagagiolo-Bardi [6] for a generalization to a polytopic case as (3.9)), for every compact set $\tilde{K} \subset \bar{\Omega}$ there exist a time $\tau_{\tilde{K}}>0$ and a constant $\beta_{\tilde{K}}>0$ (both depending only on $\tilde{K}$ and on $f$ via (3.16)) such that, for every $x \in \tilde{K}$ and for every $\alpha \in \mathcal{A}$, there exists $\bar{\alpha} \in \mathcal{A}$ such that

$$
\begin{aligned}
& \bar{y}_{x}(t) \in \bar{\Omega}, \quad \forall 0 \leq t \leq \tau, \\
& \left|J_{\tau_{\tilde{K}}}(x, \bar{\alpha})-J_{\tau_{\tilde{K}}}(x, \alpha)\right| \leq \beta_{\tilde{K}} \sup _{0 \leq t \leq \tau} \operatorname{dist}\left(y_{x}(t), \bar{\Omega}\right),
\end{aligned}
$$

where $\bar{y}_{x}(\cdot)$ and $y_{x}(\cdot)$ are respectively the trajectories of (3.13) with $\bar{\alpha}$ and $\alpha$ as control and, in general, for $t \geq 0, J_{t}$ is the corresponding cost given only by the integral part of (3.14) up to the time $t$, independently whether the trajectory stays inside $\bar{\Omega}$ or not. From (6.40), and standard estimates on the trajectories, we get the following: for every compact set $\tilde{K} \subset \bar{\Omega}$ and for every $T>0$ there exists a constant $C_{\tilde{K}, T}>0$ such that, for every $x, y \in \tilde{K}$ and for every $\alpha \in \mathcal{A}$ such that $y_{y}(t) \in \bar{\Omega}$ for all $t \in[0, T]$, there exists $\bar{\alpha} \in \mathcal{A}$ such that

$$
\begin{aligned}
& \left\|\bar{y}_{x}(t)-y_{x}(t)\right\| \leq C_{\tilde{K}, T}\|x-y\| \forall t \in[0, T], \\
& \left\|\bar{y}_{x}(t)-y_{y}(t)\right\| \leq C_{\tilde{K}, T}\|x-y\| \forall t \in[0, T], \\
& \left|J_{T}(x, \bar{\alpha})-J_{T}(y, \alpha)\right| \leq C_{\tilde{K}, T}\|x-y\| .
\end{aligned}
$$

Now, from (6.41), from the reachability condition (3.17), and again from the controllability condition (3.16), and finally from standard inequalities on the trajectories, we then get the following

$$
\begin{aligned}
& \forall K \subseteq \bar{\Omega} \text { compact } \forall \delta>0 \exists C_{K, \delta}>0 \text { such that } \\
& \forall x, y \in K, \forall \alpha \in \mathcal{A}, \exists \bar{\alpha} \in \mathcal{A} \text { such that } \\
& |J(x, \bar{\alpha})-J(y, \alpha)| \leq C_{K, \delta}\|x-y\|+\delta .
\end{aligned}
$$

To obtain (6.42), take $T>0$ such that the possible remaining part of the cost $J$ in the time interval $(T,+\infty)$ is certainly less than $\delta / 2$ for every initial point $x$ and control $\alpha \in \mathcal{A}$, and let $\tilde{K}$ be a compact set in $\mathbb{R}^{n}$ containing, up to time $T$, any trajectory starting from $K$. Then construct the control $\bar{\alpha}$ by observing the trajectory $y_{y}(\cdot)$ and making $\bar{y}_{x}(\cdot)$ have suitable behavior and cost: use (3.16) to exit from $\bar{\Omega}$ if necessary; use (6.41) to remain inside $\bar{\Omega}$ if necessary; use $(3.17)$ to reach $(\partial \Omega)_{1}$ as final exit point if necessary.

From (6.42), we easily get the continuity of $v$. It is sufficient to argue by absurd and suppose that there exist a point $x \in \bar{\Omega}$, a sequence $\left\{y_{n}\right\}_{n} \subset \bar{\Omega}$ converging to $x$, and $\varepsilon>0$ such that $\left|v(x)-v\left(y_{n}\right)\right| \geq \varepsilon$ for all $n \in \mathbb{N}$.

$v$ solves (3.18). This fact may be checked by standard techniques (see Bardi-Capuzzo Dolcetta [8]).

$v$ is the unique solution of (3.18). First of all observe that by the controllability hypothesis (3.16), $v$ certainly satisfies the condition

$$
v(x) \leq \xi_{*}(x) \quad \forall x \in(\partial \Omega)_{1} \cap(\partial \Omega)_{\tilde{1}} .
$$

Let us use the following notation: for every set of indices $I \subset\{1,2, \ldots, r\}$ we define 


$$
\begin{aligned}
& (\partial \Omega)_{I}=\bigcap_{i \in I}(\partial \Omega)_{i}, \text { and, if }(\partial \Omega)_{I} \neq \emptyset, \\
& \xi_{I}:(\partial \Omega)_{I} \rightarrow \mathbb{R}, \xi_{I}(x)=\min \left\{\xi_{i}(x) \mid i \in I, x \in(\partial \Omega)_{i}\right\}, \\
& \xi^{I}:(\partial \Omega)_{I} \rightarrow \mathbb{R}, \xi^{I}(x)=\max \left\{\xi_{i}(x) \mid i \in I, x \in(\partial \Omega)_{i}\right\}
\end{aligned}
$$

We now argue as in Bardi-Capuzzo Dolcetta [8] and in particular as in Bardi-Soravia [9] (to which the reader is strongly referred for details) which treats the unbounded domain case. The only difference here is that the boundary datum is discontinuous. However, the only possible discontinuity points are points in $(\partial \Omega)_{I}$, where $I \subseteq\{1, \ldots, r\}$ has at least two elements. But there, (3.12) and (6.43) guarantee the applicability of the argument. Indeed, we may first prove a comparison result between a bounded continuous viscosity subsolution $v_{1}$ (i.e. a function satisfying the first implications in (3.7) and in (3.8)) and a bounded continuous viscosity supersolution $v_{2}$ (i.e. a function satisfying the second implications in (3.7) and in (3.8)), the latter also satisfying (6.43). A little bit more precisely, adopting the standard double variables technique and arguing as in [9], we are led to consider only the case of sequences of maxima $x_{\varepsilon}^{\beta} \in \Omega$ and minima $y_{\varepsilon}^{\beta} \in \partial \Omega$ which, for every sufficiently small $\beta>0$, converge to a suitable point $w^{\beta} \in(\partial \Omega)_{I}$, as $\varepsilon \rightarrow 0^{+}$, where $I$ is the maximal set of indices, $\tilde{I} \subseteq\{1, \ldots, r\}$, such that $w^{\beta} \in(\partial \Omega)_{\tilde{I}}$ (note that in this case we have $\xi_{I}\left(w^{\beta}\right)=\xi_{*}\left(w^{\beta}\right)$ ). We can also suppose that, for every small $\varepsilon, y_{\varepsilon}^{\beta} \in(\partial \Omega)_{I^{\prime}}$ with $I^{\prime} \subseteq I$ independent on $\varepsilon$. Moreover, by virtue of (6.43), we may consider only the case $v_{2}\left(w^{\beta}\right)<\xi_{*}\left(w^{\beta}\right)-\gamma$, where $\gamma>0$ makes the absurd hypothesis

$$
\sup _{x \in \bar{\Omega}}\left\{v_{1}(x)-v_{2}(x)\right\}=3 \gamma
$$

hold. Hence, for every $\beta>0$ sufficiently small, denoting by $\omega^{\beta}$ a modulus of continuity for $v_{1}, v_{2}$ in $\bar{\Omega} \cap B^{\beta}$ and for $\xi_{i}$ in $(\partial \Omega)_{i} \cap B^{\beta}, i=1, \ldots, r$, where $B^{\beta}$ is a suitable ball in $\mathbb{R}^{n}$ depending on $\beta$, we have

$$
\left\{\begin{array}{l}
\xi_{*}\left(w^{\beta}\right)-\gamma>v_{2}\left(w^{\beta}\right) \geq v_{2}\left(y_{\varepsilon}^{\beta}\right)-\omega^{\beta}(\varepsilon), \\
\xi_{*}\left(w^{\beta}\right)-\gamma=\xi_{I}\left(w^{\beta}\right)-\gamma \leq \xi_{I^{\prime}}\left(w^{\beta}\right)-\gamma \leq \xi_{I^{\prime}}\left(y_{\varepsilon}^{\beta}\right)-\gamma+\omega^{\beta}(\varepsilon) \leq \xi^{*}\left(y_{\varepsilon}^{\beta}\right)-\gamma+\omega(\varepsilon),
\end{array}\right.
$$

which, for $\varepsilon$ small, gives $v_{2}\left(y_{\varepsilon}^{\beta}\right)<\xi^{*}\left(y_{\varepsilon}^{\beta}\right)$. This means that the boundary condition in (3.18) implies the verification of the equation in $y_{\varepsilon}^{\beta}$, and hence, in a standard way, we get a contradiction, eventually obtaining $v_{1} \leq v_{2}$.

Now, if we take the value function $v$ as $v_{2}$ in the preceding comparison then, for every $v_{1}$ continuous and bounded viscosity solution of (3.18), we get that $v_{1} \leq v$ and that $v_{1}$ satisfies (6.43) too. Hence, changing the role, we also get $v \leq v_{1}$, which implies the uniqueness of $v$ as solution.

Remark 6.1 Since the boundary datum $\xi$ satisfies the regularity property $\left(\xi^{*}\right)_{*}=\xi_{*}$, then the uniqueness of the value function $v$ as continuous viscosity solutions may be also obtained suitably adapting the techniques for discontinuous viscosity solution of Barles-Perthame [10] and Blanc [14] (see also Bagagiolo [5]). Indeed, the general result in those articles is the fact that all the discontinuous solutions have the same lower semicontinuous envelope; since we already know that a continuous viscosity solution exists, then it obviously must be the unique continuous solution. However, it is not excluded that other discontinuous solutions exist.

Proof of Proposition 4.4. The boundedness is obvious. Let us note that $\overline{\mathcal{H}}$ consists of four disjoint connected components and hence "continuity on $\overline{\mathcal{H}}$ " means continuity on every closed connected component $\overline{\mathcal{H}}_{(w, z)}$. Hence, using the controllability hypotheses (4.19), (4.26) and 
(4.27), the proof is similar to the first part of the proof of Proposition 3.1. In particular, note that, whenever a finite time $T>0$ is fixed, then any trajectories (starting from any point of $\overline{\mathcal{H}}$ ) may switch (i.e. change connected component) only a finite number of times $N(T)$ depending only on $T$ (and on the boundedness of the dynamics $f$ ). Hence, the argumentation as in the proof of Proposition 3.1 has to be implemented only a finite number of times $N(T)$, getting then the conclusion.

\section{References}

[1] D. Angeli, E. D. Sontag: Multi-stability in monotone input/output systems, Systems Control Lett., 51 (2004), 185-202.

[2] D. Angeli, J. E. Ferrell, E. D. Sontag: Detection of multistability, and hysteresis in a large class of biological positive-feedback systems, Proc. Natl. Acad. Sci. USA, 101 (2004), 1822-1827.

[3] F. Bagagiolo: An infinite horizon optimal control problem for some switching system, Discrete Contin. Dyn. Syst. Ser. B, 1 (2001), 443-462.

[4] F. Bagagiolo: Minimum time for a hybrid system with thermostatic switchings, in "Hybrid Systems: Computation and Control", A. Bemporad, A. Bicchi, and G. Buttazzo (Eds.), pp. 32-45, Lecture Notes in Computer Sciences 4416, Springer-Verlag, Berlin 2007.

[5] F. Bagagiolo: Optimal control of finite horizon type for a multidimensional delayed switching system, Discrete Contin. Dyn. Syst. Ser. B, 5 (2005), 239-264.

[6] F. Bagagiolo, M. Bardi: Singular perturbation of a finite horizon problem with statespace constraints, SIAM J. Control Optim., 36 (1998), 2040-2060.

[7] F. Bagagiolo, D. Bauso: Objective function design for robust optimality of linear control under state-constraints and uncertainty, accepted for publications by ESAIM: Control Optimisation, and Calculus of Variations, electronic version DOI: $10.1051 / \mathrm{cocv} / 2009040$.

[8] M. Bardi, I. C. Dolcetta: Optimal Control and Viscosity Solutions of Hamilton-JacobiBellman Equations, Birkhäuser, Boston, 1997.

[9] M. Bardi, P. Soravia: A comparison result for Hamilton-Jacobi equations and applications to some differential games lacking controllability, Funkcial. Ekvac., 39 (1994), 19-43.

[10] G. Barles, B. Perthame, Discontinuous solutions of deterministic optimal stopping time problems, RAIRO Modél. Math. Anal. Numér., 21 (1987), 557-579.

[11] G. Batt, C. Belta, R. Weiss: Model checking genetic regulatory networks with parameter uncertainty, in "Hybrid Systems: Computation and Control", A. Bemporad, A. Bicchi, and G. Buttazzo (Eds.), pp. 61-75, Lecture Notes in Computer Sciences 4416, SpringerVerlag, Berlin 2007.

[12] K. Benmansour, A. Benalia, M. Djemai, J. de Leon: Hybrid control of a multicellular converter, Nonlinear Anal. Hybrid Syst., 1 (2007), 16-29.

[13] A. Bensoussan, J. L. Menaldi: Hybrid control and dynamic programming, Dynam. Contin. Discrete and Impuls. Systems, 3 (1997), 395-442.

[14] A.-P Blanc: Deterministic exit time control problem with discontinuous exit costs, SIAM J. Control Optim., 35 (1997), 399-434.

[15] S. Dharmatti, M. Ramaswamy: Hybrid control system and viscosity solutions, SIAM J. Control Optim., 44 (2005), 1259-1288. 
[16] M. Göcke: Various concepts of hysteresis applied in economics, J. Economic Surveys, 16 (2002), 167-188.

[17] S. M. Lenhart, T. I. Seidman, J. Yong: Optimal control of a bioreactor with modal switching, Math. Models Methods Appl. Sci., 11 (2001), 933-949.

[18] D. Liberzon: Switching in Systems and Control, Birkhäuser, Boston, 2003.

[19] H. M. Soner: Optimal control problems with state-space constraints I, SIAM J. Control Optim., 31 (1986), 132-146.

[20] A. Visintin: Differential Models of Hysteresis, Springer-Verlag, Berlin, 1996.

[21] H. Zhang, M. R. James: Optimal control of hybrid systems and a system of quasivariational inequalities, SIAM J. Control Optim., 45 (2006), 722-761. 